\title{
Energy-based analysis of ultrasonically assisted turning
}

\author{
G.A. Volkov ${ }^{\mathrm{a}}$, V.A. Bratov ${ }^{\mathrm{b}}$, A.A. Gruzdkov ${ }^{\mathrm{c}}$, V.I. Babitsky ${ }^{\mathrm{d}}$, Yu.V. Petrov ${ }^{\mathrm{b}}$ and V.V. Silberschmidt ${ }^{\mathrm{d}, *}$ \\ ${ }^{a}$ St.-Petersburg State University, St.-Petersburg, Russia \\ ${ }^{\mathrm{b}}$ Institute for Problems of Mechanical Engineering RAS, St.-Petersburg, Russia \\ ${ }^{\mathrm{c}}$ St.-Petersburg State Technological Institute, St.-Petersburg, Russia \\ ${ }^{\mathrm{d}}$ Wolfson School of Mechanical and Manufacturing Engineering, Loughborough University, Loughborough, Leics., \\ $L E 113 T U, U K$
}

Received 29 January 2010

Revised 12 August 2010

\begin{abstract}
The process of ultrasonically-assisted turning (UAT) is a superposition of vibration of a cutting tool on its standard movement in conventional turning (CT). The former technique has several advantages compared with the latter, one of the main being a significant decrease in the level of cutting forces. In this paper the effects observed in UAT are analysed employing ideas of dynamic fracture mechanics. The active stage of loading duration depends heavily on ultrasonic frequency and the cutting speed; he application of the fracture criterion based on the notion of incubation time makes it possible to calculate a dependence of this duration on its threshold amplitude. An estimation of energy, necessary to create a threshold pulse in the material, is made by solving the contact Hertz problem. The obtained time dependence of energy has a marked minimum. Thus, the existence of energy-efficient loading duration is demonstrated. This explains the decrease in the cutting force resulting from superimposed ultrasonic vibration. The obtained results are in agreement with experiments on ultrasonic assisted machining of aluminium and Inconel 718 alloy.
\end{abstract}

Keywords: Ultrasonically-assisted turning, fracture energy, fracture criterion

\section{Introduction}

Ultrasonic vibrations are widely employed in industry. Ultrasound is used for cleaning and degreasing of machine parts, intensification of chemical reactions, degassing and structuring of melts as well as for many types of mechanical technologies: cutting, wire drawing, etc. Exposure to ultrasonic vibration significantly affects the material behaviour as well as the technological process; as a result of ultrasonic assistance the quality of produced parts can be considerably improved. Application of ultrasonic vibrations can have a similar effect on quality and finish of created surfaces and process precision. It also makes it possible to treat high-strength materials that are hard or even impossible to machine utilizing traditional techniques.

Numerous investigations of mechanisms of material response to ultrasonic vibration have been conducted by various researchers. For instance, it was observed that superimposing ultrasonic vibration on a tool movement in machining operations (e.g. turning, boring or drilling) resulted in a significant reduction of a force applied to the tool. Various hypotheses have been suggested to explain the change in the force as the ultrasonic vibration is applied to the tool, the main being the change of the effective deformational properties of the material or reduction of friction forces between the cutting tool and the processed material.

\footnotetext{
*Corresponding author: V.V. Silberschmidt, Fax: +44 1509 227502; E-mail: V.Silberschmidt@lboro.ac.uk.
} 


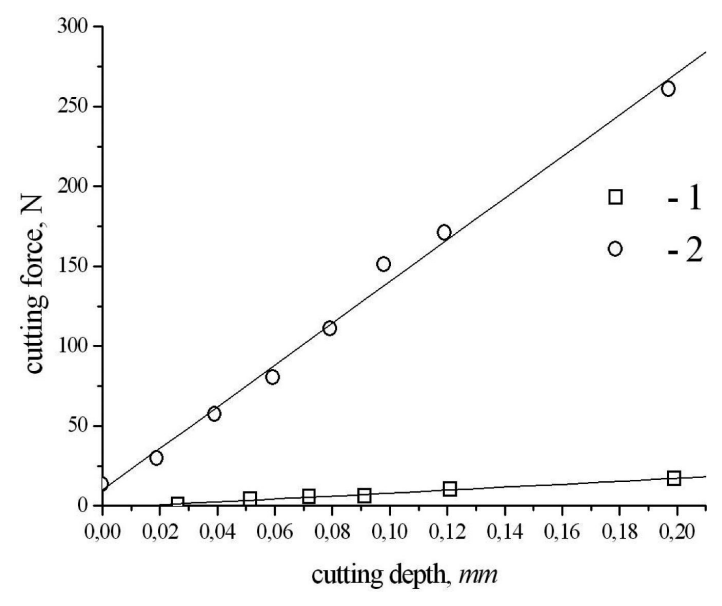

Fig. 1. Effect of depth of cut on cutting force in traditional (1) and vibration-assisted cutting (2) (after [1]).

Extensive experimental investigations of processes accompanying ultrasonic and traditional cutting were conducted in Japan [1]. Analyzing the experimental data, Kumabe [1] explained some of the observed effects of ultrasonic vibration-assisted cutting by special features of the cutter trajectory. The experiments were performed with a special experimental setup that allowed precise measurements of vibration frequency and amplitude of the cutting tool. It was also possible to register the vibration history and to measure normal and main components of the cutting force. On completion of an experiment, damage of the cutting edge of the cutter and roughness of the created surface were assessed.

It was shown that addition of vibration in normal and axial directions had no practical use apart from certain reduction in the surface roughness. Therefore, a special attention was drawn to analysis of machining assisted by vibration of the tool in the direction of the principal component of the cutting force. The typical results for this case are presented in Fig. 1. The obtained dependencies of the level of cutting force on the depth of cut are very close to linear.

It was concluded that for a wide range of materials and for arbitrary cutting depth the force produced on the cutting tool in the case of vibration-assisted cutting is significantly less as compared to traditional cutting.

The effect of the vibration amplitude on the cutting force was studied for different materials, including aluminium and carbon steel using orthogonal turning experiments. In those experiments, ultrasonic vibration with various parameters was superimposed on a constant-velocity movement of the cutting tool. The level of cutting force was used as basis for comparison of obtained results. For a low feeding rate of $0.2 \mathrm{~m} / \mathrm{min}$ an increase in the amplitude of ultrasonic vibration from $0 \mu \mathrm{m}$ to $9-15 \mu \mathrm{m}$ was accompanied by a significant reduction in the level of cutting forces - by 5-15 times. It was also found that introduction of ultrasonic vibration improved the surface finish. The test on steel were performed with frequency of $20 \mathrm{kHz}$, the frequency used to machine aluminium was $18.4 \mathrm{kHz}$. The similar improvements were found for other frequencies (up to $40 \mathrm{kHz}$ ) [1].

In experiments with aluminium samples, the effect of feed rate on the cutting force was also studied [1]. It was found that the increasing feeding rate reduced the effect of vibration on the cutting force. It was demonstrated that there existed a definite critical (threshold) feeding rate, above which the level of cutting force did not change with the varying feeding rate. It means that in the case of turning with rates exceeding the threshold rate, the main component of the cutting force is the same as for traditional cutting, i.e. vibration is not affecting the process in this case.

The effect of frequency of vibration was also analysed in turning aluminium for different feeding rates. For all the studied frequencies, the cutting force increased with the feeding rate, saturated at the plateau corresponding to the level observed in traditional cutting experiments (without vibration). At the same time, the critical feeding rate was different for different magnitudes of frequency. Comparing measured critical rates, evaluated for different levels of vibration amplitude and frequency, it was found that the critical rate is proportional to the product of vibration frequency and vibration amplitude. Thereby, the force created on the cutter in the case of vibration cutting is proportional to vibration rate and the feeding rate. 


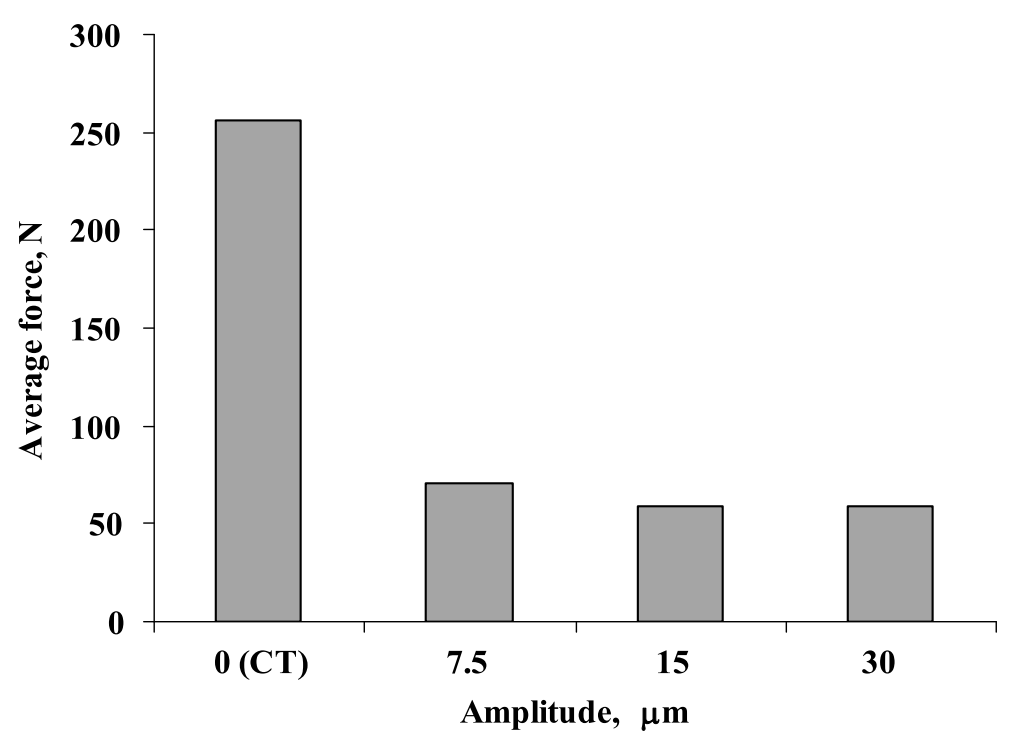

Fig. 2. Effect of frequency on cutting force in dry turning of Inconel 718 (cutting speed $335.24 \mathrm{~mm} / \mathrm{s}$; feed rate $0.2 \mathrm{~mm}$ ).

Interesting observations were made studying chips. It was found that chips produced using vibration-assisted cutting were much longer as compared to those produced with traditional cutting, they were also thinner. The character of chips also changed: in traditional cutting chips were more serrated. Measurements of their stiffness revealed that chips produced without vibration were more hardened - $196 \mathrm{MPa}$ instead of $132 \mathrm{MPa}$ for ultrasonicallyassisted turning [1]. It is an obvious sign of the lower invasiveness of the latter technique. This is also supported by analysis of residual stresses in the sub-surface layers for two machining technologies; conventional turning resulted in a higher level of such stresses.

The main reason for differences between the two cutting techniques is the intermittent character of the contact between the vibrating tool and machined material in UAT that can be called a vibro-impact contact. Since exited vibration of the tool is in the ultrasonic range, the duration of each impact is of the order of 10 microseconds. With vibration frequency being linked to duration of a single impact it is reasonable to suppose that there should exist an optimal frequency magnitude minimizing the energy necessary to initiate separation of the machined material. In other words, there should exist an optimal time of contact between the tool and the turned material, resulting in the most efficient cutting conditions. This paper aims at determining such optimal conditions, treating material separation as a type of a fracture process, and is focused on the application of the dynamic fracture mechanics concept to defining the optimum impact parameters.

\section{Finite-element modelling of tool-workpiece interaction}

The finite-element method (FEM) is one of the main approaches used to model interaction of a cutting tool and material in machining problems. Traditionally, FEM was utilized to model conventional cutting. Due to complexity of the geometry of the process zone and kinematics of the chip-formation process, 3D models accounting for a three-dimensional character of cutting forces, stress-strain and temperature fields are required [2]. Generally, in computational schemes it is assumed that chip separation occurs along some pre-defined surfaces. This condition limits the volume of material to be described. Opposite to this, in several computational schemes another mechanism of creation of a new surface was used [3], based on penetration of the tool into the machined material. Finite-element analysis should also account for coupling of thermal and mechanical processes in the cutting area [4].

A first 2D FE model of the orthogonal ultrasonically-assisted turning was developed recently to study the material behaviour and chip formation processes and to analyse a stress-strain state in the cutting zone [5]. In that model a single cycle of ultrasonic vibration in UAT of Ni-base alloy Inconel 718 was analysed in details numerically. In the 
subsequent works (e.g. [6,7]) the model was advanced to describe conventional and ultrasonically-assisted cutting in $3 \mathrm{D}$ cases. As a result of those simulations, a significant reduction of the force produced on the cutting tool in the case of ultrasonic assisted cutting was demonstrated. The effect of the amplitude of ultrasonic vibration on the level of the cutting force obtained in 3D finite-element simulations of turning of Inconel 718 is presented in Fig. 2. Apparently, there is a sharp decrease in the force at changing from the conventional technique (CT) to vibration-assisted one. To achieve comparability, the forces were averaged over time due to their intermittent character in the UAT: the tool is in contact with the chip only a part of the vibration cycle. The latter is affected by vibration parameters. The results of numerical simulations were in good agreement with experimental measurements performed at the special UAT set-up at Loughborough University, UK.

\section{Minimization of fracture energy}

Most researchers dealing with problems of dynamic fracture are using fracture criteria based on extrapolation of quasi-static fracture criteria to dynamic conditions. Though they normally account for inertia and temporal characteristics of the load applied, temporal characteristics of the fracture process are usually not taken into consideration. Utilizing this kind of approaches it is impossible to develop a method predicting a critical situation, leading to fracture, applicable to both dynamic (high-rate loads) and quasi-static cases. In this work, an incubation time fracture criterion [8], overcoming this shortcoming, is used in order to predict fracture.

This approach is based on a concept of characteristic time (known as incubation time) of a fracture process. The important feature of the incubation time fracture criterion is its capability to predict fracture initiation conditions with reliability and precision for quasi-static cases of relatively slowly changing loads and geometry as well as for dynamic cases of high-rate loads and fast changes in geometry (see, e.g. [9-13]). Moreover, the criterion is supplying a smooth transition between these two cases [11]. Hence, this approach can be applied for an arbitrary time scale of the problem - the criterion gives correct predictions in a wide range of loading rates from static problems to the extremely dynamic ones. For the present analysis we need to consider a wide range of loading rates and load durations, characteristic for two types of machining techniques. In this regard the incubation time fracture criterion is providing a unique possibility to achieve correct estimations of conditions leading to fracture for the complex problem of vibration-assisted machining. Application of fracture mechanics in analysis of material separation processes in cutting has been used by many researchers as an alternative to the use of approaches based only on plastic flow (onset of yielding). Obviously, different materials behave differently under various cutting conditions, with some demonstrating a formation of a pre-crack near the tip of a cutting tool. A quick-stop device (see for instance [14]) can provide evidence on the mechanisms in the process zone. Unfortunately, its use for UAT conditions is hardly feasible due to high frequency and low amplitude of vibrations. It is also well known that an increase in the strain rate results in increasing material's brittleness. Hence, the fracture mechanics-based concept can be considered for application to UAT that demonstrates strain rates higher by more than one order of magnitude that those in CT.

In the simplest case the incubation time fracture criterion is [8]:

$$
\frac{1}{\tau} \int_{t-\tau}^{t} \frac{\sigma(s)}{\sigma_{c}} d s \leqslant 1,
$$

where $\sigma_{\mathrm{c}}$ is tensile strength of the material, evaluated for quasi-static conditions, and is the incubation time of the fracture process. Suppose that the shape of a loading pulse can be approximated by a smooth function:

$$
\omega(t)=\left\{\begin{array}{l}
\exp \left(\frac{1}{1-\left(\frac{2 t}{t_{0}}-1\right)^{-2}}\right), \quad\left|t-\frac{t_{0}}{2}\right| \leqslant \frac{t_{0}}{2}, \\
0, \quad\left|t-\frac{t_{0}}{2}\right|>\frac{t_{0}}{2}
\end{array}\right.
$$

where $t_{0}$ is the load duration. Then the load is given by

$$
\sigma(t)=\sigma_{\max } \cdot \omega(t)
$$


where $\sigma_{\max }$ is the load amplitude. Substituting Eq. (2) into fracture criterion (1) one can obtain the critical amplitude $\sigma_{\max }$ leading to fracture and corresponding to equality in Eq. (1):

$$
\sigma_{\max }=\frac{\sigma \cdot \tau}{\max _{t \in\left[0 ; t_{0}\right]} \int_{t-\tau}^{t} \omega(s) d s} .
$$

\section{Solution of a model problem}

As an option for the way the energy is delivered to the fracture zone consider a problem of impact interaction. In [15] a problem for spherical particle with radius $R$ and velocity $V$ impacting an elastic half-space was analyzed using the classical Hertz contact scheme. Maximum stresses appearing in the half-space and duration of interaction between the particle and the half-space were calculated. According to the Hertz hypothesis the contact force $P$ arising between the particle and the half space can be presented as:

$$
\begin{aligned}
& P(t)=k h^{\frac{3}{2}}, \\
& k=\frac{4}{3} \sqrt{R} \frac{E}{\left(1-\nu^{2}\right)},
\end{aligned}
$$

where $h$ is a particle penetration; $E$ and $\nu$ are the Young's modulus and Poisson's ratio of the elastic media. The maximum penetration depth $h_{0}$ can be found as:

$$
h_{0}=\left(\frac{5 m k V^{2}}{4 k}\right)^{\frac{2}{5}}
$$

where $m$ is a particle's mass. The impact duration can be presented as

$$
t_{0}=\frac{2 h_{0}}{V} \int_{0}^{1} \frac{d \gamma}{1-\gamma^{\frac{5}{2}}}=2,94 \frac{h_{0}}{V} .
$$

The dependence of penetration $h(t)$ on time can be approximated by

$$
h(t)=h_{0} \sin \left(\frac{\pi \cdot t}{t_{0}}\right) .
$$

Time-dependent maximum tensile stress generated in the impacted media can be estimated by

$$
\sigma(V, R, t)=\frac{1-2 \nu}{2} \cdot \frac{P(t)}{\pi a^{2}(t)}
$$

where the radius of the contact area $a(t)$ is given by:

$$
a(t)=\left(3 P(t)\left(1-\nu^{2}\right) \frac{R}{4 E}\right)^{\frac{1}{3}} .
$$

Knowing the duration and amplitude of the applied load, the mass and velocity of the impacting particle can be found from Eqs (3)-(8):

$$
\begin{aligned}
& R=\frac{t_{0}}{2.94}\left(\frac{6}{5} \frac{\sigma_{\max }}{\rho(1-2 \nu)}\right)^{\frac{1}{2}} \\
& V=\left(\frac{5}{4} \frac{\rho \pi\left(1-\nu^{2}\right)}{E}\right)^{2}\left(\frac{6}{5} \frac{\sigma_{\max }}{\rho(1-2 \nu)}\right)^{\frac{5}{2}},
\end{aligned}
$$




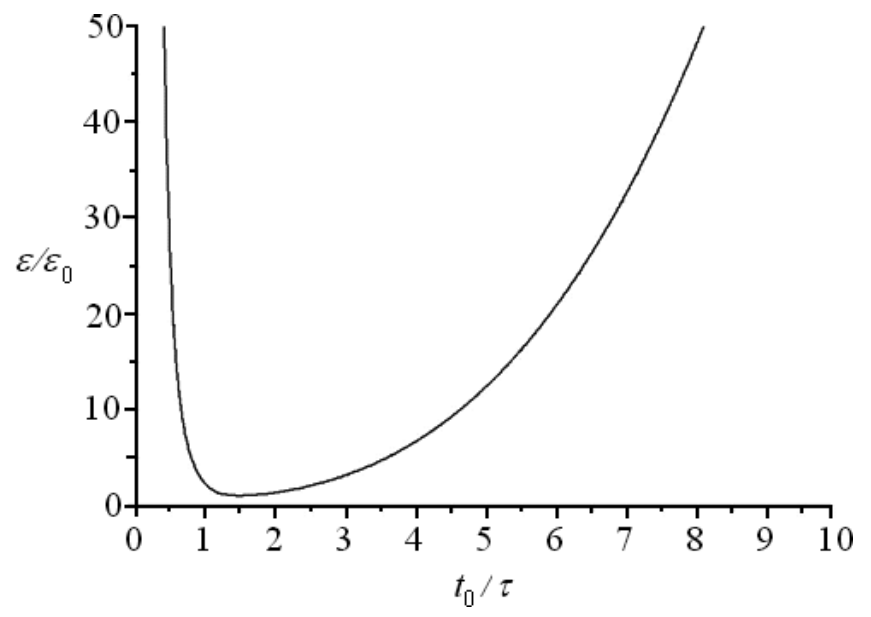

Fig. 3. Energy needed to initiate fracture $v s$. impact duration $\left(\sigma_{\mathrm{c}}=700 \mathrm{MPa}\right.$ and $\left.\tau=8 \mu \mathrm{s}\right)$.

where $\rho$ is a parameter of load intensity, in units of mass density, and $\sigma_{\max }$ is the maximum stress (i.e. load amplitude). Evaluating initial kinetic energy of the spherical particle one can estimate energy, required to create fracture in the impacted media:

$$
\varepsilon=\alpha \cdot \frac{t_{0}^{3} \sigma_{\max }^{\frac{13}{2}}}{\rho^{\frac{3}{2}} E^{4}},
$$

where

$$
\alpha=\frac{2}{3} \frac{\pi^{5}}{(2.94)^{3}}\left(\frac{5\left(1-\nu^{2}\right)}{4}\right)^{4}\left(\frac{6}{5(1-2 \nu)}\right)^{\frac{13}{2}}
$$

is a dimensionless coefficient. This energy is plotted as a function of impact duration in Fig. 3 in dimensionless coordinates. Material's properties are taken to be equal to properties of Inconel 718 alloy $\left(E=204.9 \cdot 10^{9} \mathrm{~N} / \mathrm{m}^{2}\right.$, $\nu=0.284, \sigma_{\mathrm{c}}=0.7 \cdot 10^{9} \mathrm{~N} / \mathrm{m}^{2}$ and $\left.\tau=8 \mu \mathrm{s}\right)$. A choice of this material was determined by its extensive experimental studies in conditional and ultrasonically-assisted machining. For this material, a significant reduction of cutting force was registered as ultrasonic vibration was applied to the cutting tool (i.e. continuous loading was substituted by pulsed impact loading) [15].

The choice of the magnitude of the incubation time $\tau=8 \mu \mathrm{s}$ has the following reasons. The minimum of the energy needed to create a fracture impact is achieves for the pulse duration that is two to three times higher than the incubation time $\tau$ [12]. The oscillation frequency in experiment of ultrasonic assisted cutting of Inconel 718 equals to $20 \mathrm{kHz}$, hence the minimum impact duration is about $\frac{1}{2 f}=25 \mu \mathrm{s}$. It was suggested that the lowest power inputs corresponds to pulses with durations closest to minimum. Therefore, the incubation time is taken as one third of these impacts' average duration.

\section{Computation of average cutting force}

Suppose the force applied to the tool consisting of two components: dynamic component responsible for impactinduced material separation and a constant component $F_{0}$, related to kinematics of the cutting process. The magnitude of this constant force can be obtained from experiments. Taking into account the fact that the energy that should be supplied in order to create a fracturing loading pulse will be equal to the work of the force on displacement, equal to the vibration amplitude, the cutting force can be presented as

$$
F=\frac{\varepsilon}{\lambda}+F_{0} .
$$




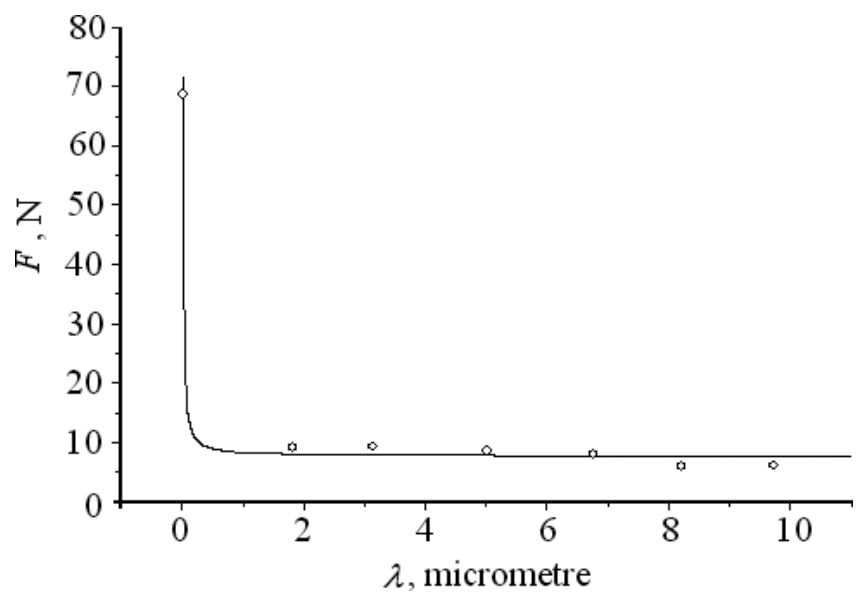

Fig. 4. Effect of ultrasonic vibration amplitude on cutting force. Comparison of calculations (solid line) and experimental data for turning of aluminium.

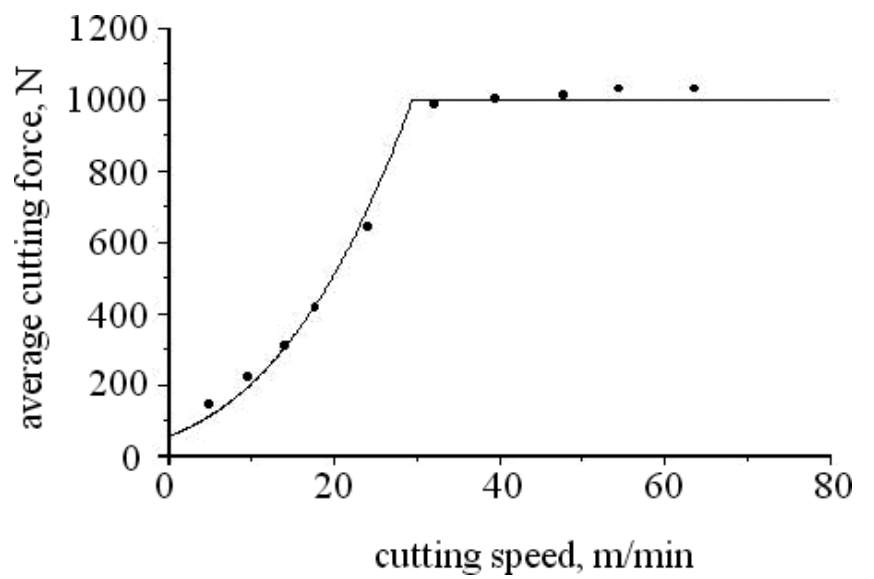

Fig. 5. Average force created on the cutter as a function of material feeding rate for ultrasonic assisted cutting of Inconel 718 alloy: $f=20 \mathrm{kHz}$, $\lambda=25 \mu \mathrm{m}$. Comparison of theoretical predictions (solid line) with results of FEM simulations.

where is the amplitude of vibrations. It should be noted that the absolute value of energy needed to create a fracturing load pulse depends on parameter $\rho$ (see Eq. (9)). The effect of amplitude on the cutting force can be calculated using Eq. (10) (Fig. 4); it is in good agreement with experimental measurements [1]. In these calculations, $F_{0} \approx 10 \mathrm{~N}$ and the cutting depth is equal to $0.05 \mathrm{~mm}$.

Increase in the material feeding rate will result in the growing duration of contact between the tool and machined material (i.e. loading duration will increase). Therefore, as the critical level $\omega$ will be achieved, the loading duration will reach some threshold value $t_{0}^{*}$, above which the material will respond to the load in a quasi-static way [16,17]. Thus, for feeding rates exceeding the critical level, the material's response will be the same as for traditional cutting. To obtain the loading duration as a function of feeding rate, the following formula can be suggested:

$$
t_{0}=\frac{1}{2 \nu}+\frac{\omega}{\omega_{\mathrm{c}}} \cdot\left(t_{0}^{*}-\frac{1}{2 \nu}\right)
$$

Using Eqs (10) and (11) it is possible to plot the average force applied to the tool as a function of the feeding rate for ultrasonically-assisted cutting of Inconel 718 alloy [7] (see Fig. 5). Similar dependencies are presented in Fig. 6 to compare the calculated results for ultrasonic turning of aluminium $\left(\tau=12 \mu \mathrm{s}, \sigma_{\mathrm{c}}=0.1 \cdot 10^{9} \mathrm{~Pa}\right)$ with available experimental measurements [1]. 


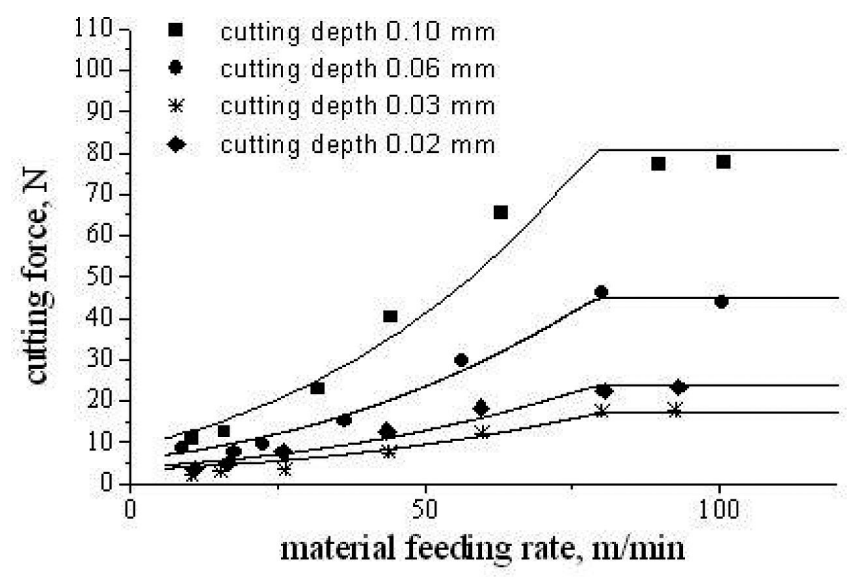

Fig. 6. Effect of material feeding rate on average cutting force as a function of for ultrasonically- assisted cutting of aluminium; $f=20 \mathrm{kHz}$, $\lambda=10 \mu \mathrm{m}, F_{0}=3 \mathrm{~N}$. Comparison of calculations (solid line) and experimental data.

The level of cutting force in the case of quasi-static load, that depends on the cutting depth, is defined by the value of $\rho$. The duration of pulsed interaction is inversely proportional to the ultrasonic vibration frequency. Therefore, knowing dynamic strength properties of the machined metal it is possible to predict optimal operational parameters for the machining device.

\section{Conclusions}

The obtained results, based on the incubation-time concept and employing methods of fracture mechanics, provide an explanation to the behaviour of the force applied to the cutting tool in case of ultrasonically-assisted machining. The approach allowed a demonstration for the existence of optimal energy-saving regimes of metal separation in machining. It is shown that such a regime is linked to a temporal character of the fracture process. Optimal energy saving for the determined durations of the critical impact loads provides a possibility to explain a significant drop in the force generated on the cutting tool in the case of ultrasonically-assisted machining. Using the developed model it is also possible to assess the effect of the feed rate on the cutting force.

\section{References}

[1] J. Kumabe, Vibration Cutting - Fundamentals and Applications, Jikkyo Shuppan, (in Japanese), 1979.

[2] E. Ceretti, M. Lucchi and T. Altan, FEM simulation of orthogonal cutting: serrated chip formation, J Mater Process Technol 95(1) (1999), 17-26.

[3] o. Pantale, J.-L. Bacaria, O. Dalverny, R. Rakotomalala and S. Caperaa, 2d and 3d numerical models of metal cutting with damage effects, Comput Meth Appl Mech Eng 193(39-41) (2004), 4383-4399.

[4] M. Ramesh, K.N. Seetharamu, N. Ganesan and G. Kuppuswamy, Finite element modelling of heat transfer analysis in machining of isotropic materials, Int J Heat Mass Transf 42(31) (1999), 458-472.

[5] A. Mitrofanov, V. Babitsky and V. Silberschmidt, Finite element simulations of ultrasonically assisted turning, Comput Mater Sci 28 (2003), 645-653

[6] A. Mitrofanov, V. Babitsky and V. Silberschmidt, Finite element modelling of ultrasonically assisted turning of Inconel 718, J Mater Process Technol 153-154 (2004), 233-239.

[7] N. Ahmed, A.V. Mitrofanov, V.I. Babitsky and V.V. Silberschmidt, 3D finite element analysis of ultrasonically assisted turning, Comput Mater Sci 39 (2007), 149-154.

[8] N. Morozov and Y. Petrov, Dynamics of Fracture, Springer-Verlag, Berlin, 2000.

[9] Y.V. Petrov and N.F. Morozov, On the modeling of fracture of brittle solids, J Appl Mech 61 (1994), $710-712$.

[10] Y.V. Petrov and E.V. Sitnikova, Dynamic cracking resistance of structural materials predicted from impact fracture of an aircraft alloy, Tech Phys 49(1) (2007), 57-60.

[11] V. Bratov and Y. Petrov, Application of incubation time approach to simulate dynamic crack propagation, Int J Fract 146 (2007), 53-60. 
[12] V. Bratov and Y. Petrov, Optimizing energy input for fracture by analysis of the energy required to initiate dynamic mode I crack growth, Int J Solid Struct 44 (2007), 2371-2380.

[13] V.A. Bratov, N.F. Morozov and Y.V. Petrov, Simulation of SMART1 Satellite Impacting the Moon Surface, Dokl Phys 419(1) (2008), $54-58$.

[14] J. Ellis, R. Kirk and G. Barrow, The development of a quick-stop device for metal cutting research, Int J Machine Tool Design Res (3) (1969), 321-339.

[15] K.L. Johnson, Contact Mechanics, Cambridge University Press, 1987.

[16] Yu.V. Petrov, Incubation time criterion and the pulsed strength of continua: Fracture, cavitation, and electrical breakdown, Dokl Phys 49(4) (2004), 246-249.

[17] V.A. Bratov, N.F. Morozov and Yu.V. Petrov, Dynamic Strength of Continuum. St. Petersburg University Press, 2009. 

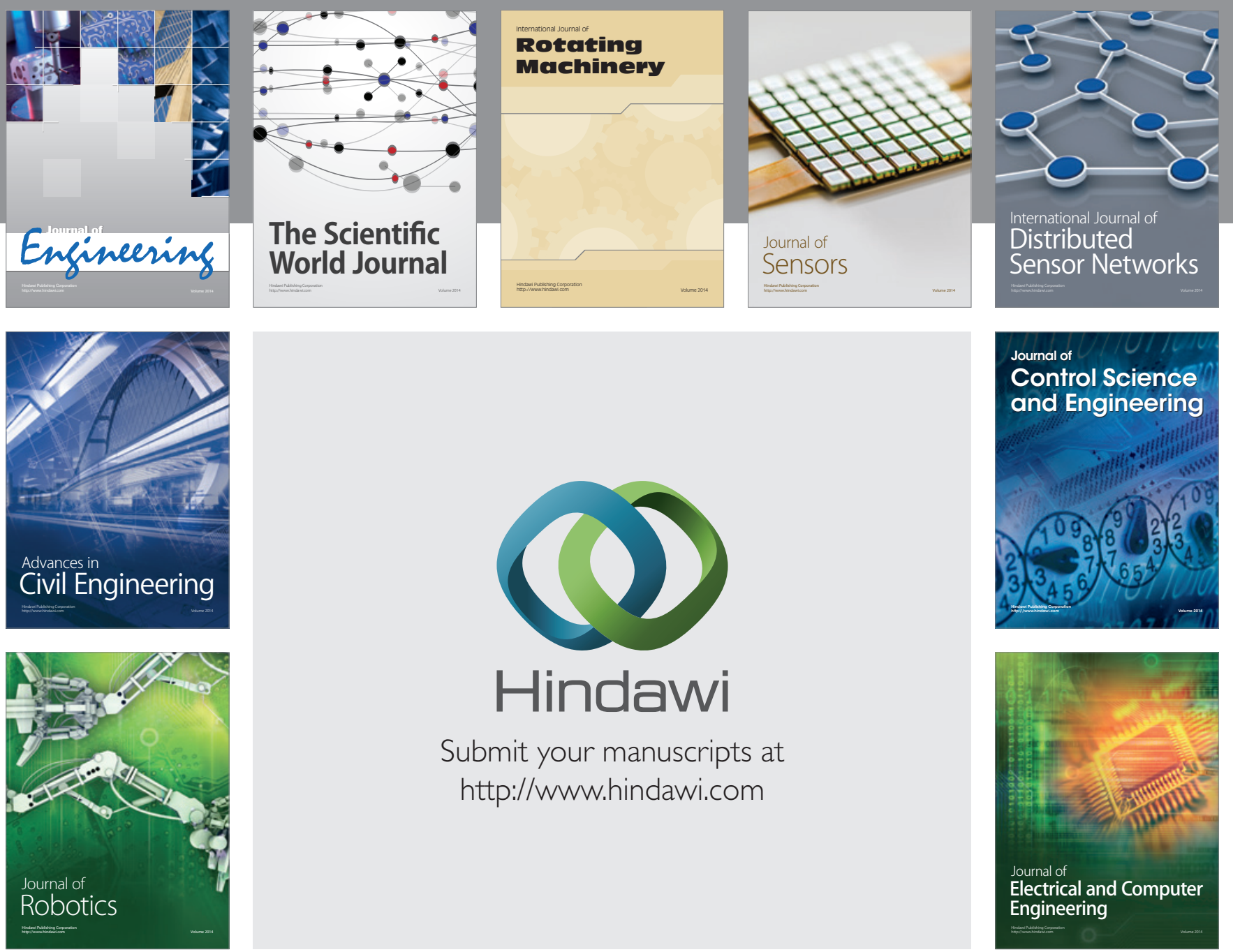

Submit your manuscripts at

http://www.hindawi.com
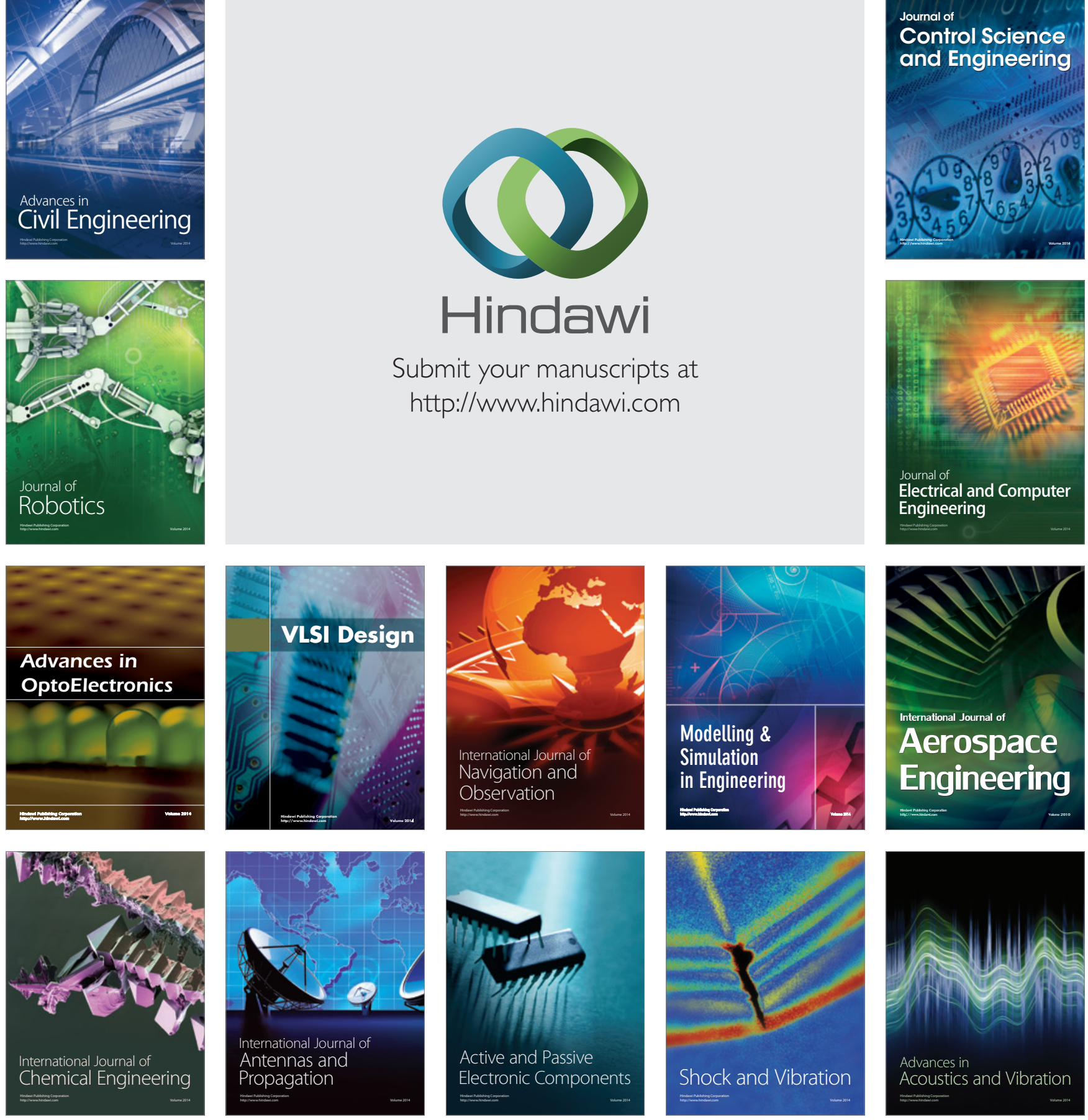